\title{
Erratum to: Daphnia revisited: local stability and bifurcation theory for physiologically structured population models explained by way of an example
}

\author{
Odo Diekmann' ${ }^{1}$ Mats Gyllenberg ${ }^{2}$ - J. A. J. Metz Me, 3,4 $^{\text {. }}$ \\ Shinji Nakaoka ${ }^{5}$ - André M. de Roos ${ }^{6}$
}

Published online: 19 June 2017

(C) Springer-Verlag Berlin Heidelberg 2017

\section{Erratum to: J. Math. Biol. (2010) 61:277-318 DOI 10.1007/s00285-009-0299-y}

In the original publication, the addresses of the authors Dr. J.A.J. Metz and Dr. S. Nakaoka were incorrectly published. The correct address list for the authors are:

J.A.J. Metz: Institute of Biology and Institute of Mathematics, Leiden University, P.O. Box 9516, 2300RA Leiden, The Netherlands. S. Nakaoka: Graduate School of

The online version of the original article can be found under doi:10.1007/s00285-009-0299-y.

Shinji Nakaoka

snakaoka@m.u-tokyo.ac.jp

Odo Diekmann

O.Diekmann@uu.nl

Mats Gyllenberg

mats.gyllenberg@helsinki.fi

J. A. J. Metz

J.A.J.Metz@biology.leidenuniv.nl

André M. de Roos

A.M.deRoos@uva.nl

1 Department of Mathematics, University of Utrecht, P.O. Box 80010, 3508 TA Utrecht, The Netherlands

2 Department of Mathematics and Statistics, University of Helsinki, P.O. Box 68, 00014 Helsinki, Finland

3 Institute of Biology and Institute of Mathematics, Leiden University, P.O. Box 9516, 2300 RA Leiden, The Netherlands

4 Evolution and Ecology Program, International Institute of Applied Systems Analysis, 2361 Laxenburg, Austria 
Medicine, University of Tokyo, 7-3-1 Hongo, Bunkyo-ku, Tokyo 113-0033, Japan. E-mail: snakaoka@m.u-tokyo.ac.jp.

While working on their paper (Breda et al. 2015), Philipp Getto and Julia Sánchez Sanz found that formula (4.22) is incomplete (indeed, the solution operator of the homogeneous version of the linearized equation (4.3) should act on the second term at the right hand side of (4.22)). As a consequence (4.23), (4.24), (4.25), (4.31) and (4.32) are incorrect as well. Actually, as it turned out, there were additional inaccuracies. The correct formulas are

$$
\begin{aligned}
D_{3} \xi(\tau ; a, \bar{S}) \psi= & \eta(\tau)+e^{\int_{\bar{\tau}}^{\tau} \frac{\partial g}{\partial \xi}(\bar{\xi}(\sigma), \bar{S}) d \sigma}\left(\frac{g\left(\xi_{A}+, \bar{S}\right)}{g\left(\xi_{A}-, \bar{S}\right)}-1\right) \eta(\bar{\tau}) \\
D_{3} \xi(\tau ; a, \bar{S}) \psi= & \eta(\tau)+e^{\int_{\bar{\tau}}^{\tau} \frac{\partial g}{\partial \xi}(\bar{\xi}(\sigma), \bar{S}) d \sigma}\left(\frac{g\left(\xi_{A}+, \bar{S}\right)}{g\left(\xi_{A}-, \bar{S}\right)}-1\right) \eta(\bar{\tau}) H(\tau-\bar{\tau}) \\
D_{2} \Xi(a ; \bar{S}) \psi= & \int_{0}^{a} K(a, \alpha) \psi(-a+\alpha) d \alpha \\
& +\left(\frac{g\left(\xi_{A}+, \bar{S}\right)}{g\left(\xi_{A}-, \bar{S}\right)}-1\right) \int_{0}^{\bar{\tau}} K(a, \alpha) \psi(-a+\alpha) d \alpha \cdot H(a-\bar{\tau}) . \\
D_{2} \mathcal{F}(a, \bar{S}) \psi= & \int_{0}^{a} L(a, \theta) \psi(-a+\theta) d \theta-\frac{\mu\left(\xi_{A}+, \bar{S}\right)-\mu\left(\xi_{A}-, \bar{S}\right)}{g\left(\xi_{A}-, \bar{S}\right)} \\
& \times \int_{0}^{\bar{\tau}} K(a) \\
& -\left(\frac{g\left(\xi_{A}+, \bar{S}\right)}{g\left(\xi_{A}-, \bar{S}\right)}-1\right) \overline{\mathcal{F}}(a) \\
& \times \int_{0}^{\bar{\tau}} \int_{\bar{\tau}}^{a} \frac{\partial \mu}{\partial \xi}(\bar{\xi}(\sigma), \bar{S}) K(\sigma, \alpha) d \sigma \psi(-a+\alpha) d \alpha H(a-\bar{\tau}) .
\end{aligned}
$$

$$
\begin{aligned}
k_{12}(a)= & \bar{b} \int_{\max \{0, \bar{\tau}-a\}}^{\infty}\{\beta(\bar{\xi}(a+\theta), \bar{S}) L(a+\theta, \theta) \\
& \left.+\frac{\partial \beta}{\partial \xi}(\bar{\xi}(a+\theta), \bar{S}) K(a+\theta, \theta) \overline{\mathcal{F}}(a+\theta)\right\} d \theta \\
& +\bar{b}\left(\frac{g\left(\xi_{A}+, \bar{S}\right)}{g\left(\xi_{A}-, \bar{S}\right)}-1\right) \int_{\max \{0, \bar{\tau}-a\}}^{\bar{\tau}} K(a+\theta, \theta) \\
& \times \frac{\partial \beta}{\partial \xi}(\bar{\xi}(a+\theta), \bar{S}) \overline{\mathcal{F}}(a+\theta) d \theta
\end{aligned}
$$

5 Graduate School of Medicine, University of Tokyo, 7-3-1 Hongo, Bunkyo-ku, Tokyo 113-0033, Japan

6 Institute for Biodiversity and Ecosystem Dynamics, University of Amsterdam, P.O. Box 94248, 1090 GE, Amsterdam The Netherlands 


$$
\begin{aligned}
+ & \bar{b} \frac{\mu\left(\xi_{A}-, \bar{S}\right)-\mu\left(\xi_{A}+, \bar{S}\right)}{g\left(\xi_{A}-, \bar{S}\right)} \int_{\max \{0, \bar{\tau}-a\}}^{\bar{\tau}} \beta(\bar{\xi}(a+\theta), \bar{S}) \\
& \times \overline{\mathcal{F}}(a+\theta) K(\bar{\tau}, \theta) d \theta-\bar{b}\left(\frac{g\left(\xi_{A}+, \bar{S}\right)}{g\left(\xi_{A}-, \bar{S}\right)}-1\right) \\
& \times \int_{\max \{0, \bar{\tau}-a\}}^{\bar{\tau}} \beta(\bar{\xi}(a+\theta), \bar{S}) \overline{\mathcal{F}}(a+\theta) \int_{\bar{\tau}}^{a+\theta} \frac{\partial \mu}{\partial \xi}(\bar{\xi}(\sigma), \bar{S}) K(\sigma, \theta) d \sigma d \theta \\
& +\chi[0, \bar{\tau}](a) \bar{b} \frac{\beta\left(\xi_{A}+, \bar{S}\right) \overline{\mathcal{F}}(\bar{\tau})}{g\left(\xi_{A}-, \bar{S}\right)} K(\bar{\tau}, \bar{\tau}-a) . \\
k_{22}(a)= & -\bar{b} \int_{0}^{\infty}\{\gamma(\bar{\xi}(a+\theta), \bar{S}) L(a+\theta, \theta) \\
& \left.+\frac{\partial \gamma}{\partial \xi}(\bar{\xi}(a+\theta), \bar{S}) K(a+\theta, \theta) \overline{\mathcal{F}}(a+\theta)\right\} d \theta \\
& -\bar{b}\left(\frac{g\left(\xi_{A}+, \bar{S}\right)}{g\left(\xi_{A}-, \bar{S}\right)}-1\right) \int_{\max \{0, \bar{\tau}-a\}}^{\bar{\tau}} K(a+\theta, \theta) \frac{\partial \gamma}{\partial \xi}(\bar{\xi}(a+\theta), \bar{S}) \overline{\mathcal{F}}(a+\theta) d \theta \\
& -\bar{b} \frac{\mu\left(\xi_{A}-, \bar{S}\right)-\mu\left(\xi_{A}+, \bar{S}\right)}{g\left(\xi_{A}-, \bar{S}\right)} \int_{\max \{0, \bar{\tau}-a\}}^{\bar{\tau}} \gamma(\bar{\xi}(a+\theta), \bar{S}) \overline{\mathcal{F}}(a+\theta) K(\bar{\tau}, \theta) d \theta \\
& +\bar{b}\left(\frac{g\left(\xi_{A}+, \bar{S}\right)}{g\left(\xi_{A}-, \bar{S}\right)}-1\right) \int_{\max \{0, \bar{\tau}-a\}}^{\bar{\tau}} \gamma(\bar{\xi}(a+\theta), \bar{S}) \overline{\mathcal{F}}(a+\theta) \\
& \times \int_{\bar{\tau}}^{a+\theta} \frac{\partial \mu}{\partial \xi}(\bar{\xi}(\sigma), \bar{S}) K(\sigma, \theta) d \sigma d \theta \\
& -\chi[0, \bar{\tau}](a) \bar{b} \frac{\left(\gamma\left(\xi_{A}+, \bar{S}\right)-\gamma\left(\xi_{A}-, \bar{S}\right)\right)}{g\left(\xi_{A}-, \overline{\mathcal{F}}\right)}(\bar{\tau}) K(\bar{\tau}, \bar{\tau}-a) . \\
& \\
& \\
&
\end{aligned}
$$

We thank Philipp Getto and Julia Sánchez Sanz for discovering the mistake and pointing it out to us. We also thank Francesca Scarabel for checking the correctness of the formulas above. Moreover, we are very happy with the continuation of our work in Breda et al. (2015).

\section{References}

Breda D, Getto P, Sánchez Sanz J, Vermiglio R (2015) Computing the eigenvalues of realistic Daphnia models by pseudospecral methods. SIAM J Sci Comput 37:A2607-A2629 